\title{
Lutz Winckler
}

\section{Gedächtnis des Exils: Erinnerung als Rekonstruktion} Vorwort

Die Frage nach dem Gedächtnis des Exils bedeutet mehr als eine Erweiterung des Themenspektrums der Forschung, sie verweist auf einen Einschnitt im historischen Bewusstsein und der wissenschaftlichen Auseinandersetzung mit dem Exil: auf die Tatsache, dass das Exil zwischen 1933 und 1945 mit dem Tod der Zeitzeugen und Zeitgenossen zur Geschichte wird, deren Beziehung und Bedeutung für die Gegenwart erst rekonstruiert werden muss. Dies ist die Aufgabe des Gedächtnisses und der Erinnerung. Gedächtnis und Erinnerung sind komplementär: "Das Gedächtnis", so Aleida Assmann, wist die Dispositionsmasse, aus der die Erinnerung auswählt, aktualisiert, sich bedient " ${ }^{1}$. Um diesen Zusammenhang zu erläutern, soll zunächst ein Blick auf die unterschiedlichen Formen des Gedächtnisses geworfen werden.

Aleida Assmann unterscheidet zwei Formen des Gedächtnisses: das kommunikative Gedächtnis und das kulturelle Gedächtnis. Träger des kommunikativen Gedächtnisses sind die Mitlebenden; seine zeitliche Grenze umschließt drei Generationen. Sein Inhalt umfasst einen überschaubaren, aber wechselnden Interpretationen unterliegenden zeitgenössischen Lebens- und Erfahrungszusammenhang. Das »kommunikative Gedächtnis«, so Aleida Assmann, mentsteht in einem Milieu räumlicher Nähe, regelmäßiger Interaktion, gemeinsamer Lebensformen und geteilter Erfahrungen ${ }^{2}$. Erinnert wird in den narrativen Formen der mündlichen Rede, des Gesprächs, biografischer und historischer Erzählungen: Das kommunikative Gedächtnis ist unabgeschlossen, fragmentarisch, labil. Diese Eigenschaften verbinden sich mit einem hohen Maß emotionaler Selbstbeteiligung und selbst- bzw. gruppenbezogener Wertung. Der emotionale Erinnerungshorizont ist immer auch ein partikularer Wertungshorizont.

Das kulturelle Gedächtnis löst sich aus dem Lebenszusammenhang der Zeitgenossen und ihrer auf unmittelbare Kommunikation angelegten Erinnerungsformen. Seine Träger sind Institutionen wie Bibliotheken, Archive und Museen. Kulturelle Erinnerung vermittelt sich über Medien: über die Schrift, Bilder und Objekte, über Zeichen und Symbole. Der Zeithorizont ist potenziell unbegrenzt, er reicht über den individuellen und generationellen Zeitbezug hinaus auf die gesamte, dem Wissen verfügbare Geschichte. ${ }^{3}$ Lässt sich das kommunikative Gedächtnis als Gedächtnis der Zeitgenossen definieren, so ist das kulturelle Gedächtnis das Gedächtnis der Nachgebore- 
nen. Unterschiedlich ist auch das Verhältnis zur Geschichte: An die Stelle der emotionalen Nähe tritt die kritische Distanz.

Kommunikatives und kulturelles Gedächtnis haben eine gemeinsame Eigenschaft: Sie sind, wie Jan Assmann betont, "identitätskonkret ${ }^{4}$ - das heißt, sie tragen zur Identität der erinnernden Person oder der Gruppe bei, bestimmen die Normen ihres Verhaltens. Aber die Art und Weise dieses Identitätsbezugs ist verschieden: Die Unmittelbarkeit des lebensgeschichtlichen Zusammenhangs von Erinnern, Erfahren und Handeln, der für das kommunikative Gedächtnis bestimmend ist, ist im kulturellen Gedächtnis aufgehoben und muss immer neu rekonstruiert werden. Das verleiht dem kulturellen Gedächtnis und den daraus abgeleiteten Erinnerungs- und Orientierungsformen eine "irreduzible Vielstimmigkeit"s. Das kulturelle Gedächtnis ist selbstreferenziell: Der Erinnerungsvorgang wird von der Emotionalität umgestellt auf Reflexivität. ${ }^{6}$

Kommunikatives und kulturelles Gedächtnis schweben nicht im geschichtsfreien Raum. Beide entstehen und verlaufen innerhalb historischer und sozialer "Rahmen" - ein Begriff der auf Maurice Halbwachs zurückgeht. ${ }^{7}$ Halbwachs versteht darunter Artikulationsformen und Normen des kollektiven Gedächtnisses, wie sie sich aus dem Lebenszusammenhang der Individuen innerhalb sozialer Gruppen - der Familie, dem Beruf, religiösen und politischen Gemeinschaften - entwickeln. Diese sozialen Rahmen bilden den Horizont des Gedächtnisses. Das kommunikative Gedächtnis artikuliert sich innerhalb dieser Horizonte, individuelle und kollektive Identität folgen der Logik von Einschließung und Ausschluss. Im kulturellen Gedächtnis werden die Horizonte als Bedingungen der Erinnerung immer mit reflektiert, Identität geht aus dem Vergleich unterschiedlicher Horizonte hervor.

Funktionen und Formen des kommunikativen und des kulturellen $\mathrm{Ge}$ dächtnisses lassen sich durch den Vergleich mit der diskursiven Formation des "Zeugen" und des "Archivs" näher erläutern. Ich stütze mich dabei auf die Epistemologie des Zeugnisses und des Archivs, die Paul Ricœur entwickelt hat. ${ }^{8}$ Das Zeugnis ist nicht zu trennen von der Person des Zeugen: Zeugnisse - sowohl Aussagen, Texte oder Objekte - stehen in unmittelbarem Zusammenhang mit der Person des Zeugen; sie erhalten ihre Bedeutung aus dem Leben und der Geschichte des Zeugen. Entscheidend ist die Glaubwürdigkeit (fiabilité) des Zeugen, sie verbürgt die Wahrhaftigkeit des Zeugnisses. Der Zeuge ist nicht allein, er benötigt jemanden der ihn befragt, der ihm zuhört: Zeuge und Zuhörer bilden den Kern einer Gemeinschaft, die über den Zeugen hinausreichen kann - eine kommunikative Atmosphäre des Verstehens und Vertrauens (sociabilité). Auf diese Weise entstehen Zeugnisgemeinden, die die Sache des Zeugen vertreten. Diese Zeugnisgemeinden sind das diskursive Modell des kommunikativen Gedächtnisses. 
Das Archiv trennt die Zeugnisse vom Zeugen, die Objekte von der Person ihres Besitzers, Benutzers oder Herstellers. Das Archiv bewahrt "Reste" auf: Dokumente, Texte und Gegenstände; es sammelt funktions- und herrenlose Dinge und ordnet sie nach eigenen institutionell vorgegebenen Kriterien. Als Archiv im weiteren Sinn verstehen wir die diskursive Form, die die kulturelle Überlieferung annimmt, die aus dem kommunikativen Generationenzusammenhang herausfällt. Die Bedeutung der im "Archiv" überlieferten Objekte - der Gegenstände, Texte, Bilder - muss neu erschlossen werden. Die dialogische Struktur und das Vertrauensband zwischen Zeugen und Zuhörer, die Formen direkter Kommunikation werden ersetzt durch methodisch abgesicherte Interpretationsverfahren, die die Distanz zwischen dem Zeugnis und dem Interpreten überbrücken sollen. Die Interpretation stützt sich dabei auf "Spuren ", die Hinweise geben auf den Autor, Eigentümer oder Benutzer von Texten und Objekten, auf die historischen Voraussetzungen ihrer Hervorbringung, die Formen und Funktionen ihres Gebrauchs. Zugleich muss die Interpretation der Tatsache gerecht werden, dass sich der Charakter des Zeugnisses im Archiv verändert. Herausgelöst aus dem Lebenszusammenhang des Zeugen wird es zum Zeichen, zum Bestandteil eines kulturellen Bedeutungszusammenhangs von Texten und Gegenständen, dessen Deutung durch den historischen Vergleich erfolgt. ${ }^{10}$

Der Statuswechsel ${ }^{11}$ der Texte und Objekte zu Bedeutungsträgern, die den Wandel vom Zeugnis zum Zeichen begleitet, schließt auch ein verändertes Verhältnis zur Geschichte ein. Für den Zeugen existiert Geschichte nur als eigene, unvollendete Geschichte. Die Vergangenheit wird ihm zur Gegenwart, in der die Geschichte seines Lebens wieder neu beginnt. Es gibt eine traumatische Form der Wiederkehr der Vergangenheit: als Gegenwärtigkeit des dem Zeugen zugefügten Schmerzes, des erlittenen Unrechts, die nicht vergehen. Und es gibt eine utopisch aufgeladene, moralisch begründete Form der Wiederkehr der Vergangenheit: als Forderung, die Bedingungen des Überlebens für die eigene Person und für die Menschheit (wieder)herzustellen. Das Archiv vermag weniger und mehr: Es verspricht keine Wiederkehr der Vergangenheit, keine Heilung der Geschichte, keine Tilgung der Schuld. Es stellt sich die Aufgabe, aus den Resten und Spuren der gesammelten und aufbewahrten Dokumente die Geschichte ihrer Entstehung, ihrer Zerstörung und ihres Verlusts abzulesen und mithilfe der so gewonnen Daten ihre materielle Geschichte zu rekonstruieren. Ihre Bedeutung als kulturelle Zeichen erhalten die Zeugnisse im Rahmen des gegenwärtigen Erinnerungs- und Erfahrungshorizonts. Die Geschichte ist im Archiv Vergangenheit: Sie kehrt nicht wieder wie im Zeugnis, wird allenfalls durch Wissen und Vergleich vergegenwärtigt. Texte, Bilder, Gegenstände des Archivs sind Spuren einer Vergangenheit, die vergangen ist. Erinnerung, nicht Vergegenwärtigung, Eingedenken nicht Wiedergutmachung ist die dem Archiv angemessene Form 
der wissenschaftlichen Annäherung. Das moralische Interesse, das sich damit verbindet, zielt auf die öffentliche Anerkennung universalistischer Werte, in der auch die Einzelschicksale ihren Stellenwert erhalten. Diese Form der Erinnerung teilt das Archiv mit dem kulturellen Gedächtnis.

Kommunikatives und kulturelles Gedächtnis und die entsprechenden Diskurse des Zeugnisses und des Archivs sind zwei Modi der Erinnerung, die unterschiedliche Methoden der wissenschaftlichen Annäherung an die Geschichte beschreiben, aber im historischen Prozess der Exilforschung nebenund miteinander auftreten. Zeugen und Zeugnisse, deren Befragung und Auswertung, Sammlung und Dokumentation stehen am Beginn der Exilforschung ${ }^{12}$. Walter F. Berendsohns 1946 im Europa Verlag Zürich erschienene "Humanistische Front", auf die die literaturwissenschaftliche Exilforschung zurückgeht, ist das Werk eines "Zeugen". Sein bestimmender Impuls, den Opfern Gerechtigkeit widerfahren zu lassen, die vergessenen Werke und Autoren ins Leben zurückzurufen, bezeichnet eine Haltung, die die Forscher ins Archiv begleiten. Verändert hat sich nur das Verhältnis zur Geschichte: Wo der Zeuge und die Zeugenerinnerung Kontinuität voraussetzen, steht für den Forscher der Bruch mit der Vergangenheit. Distanz ersetzt die Nähe, wissenschaftliche Kritik tritt an die Stelle der evokativen Vergegenwärtigung. Die wissenschaftliche Auseinandersetzung mit der Vergangenheit nimmt die Form der Erinnerungsarbeit an.

Paul Ricœur spricht im Anschluss an Freud von der "Arbeit der Erinnerung ${ }^{13}$. Er versteht darunter einmal die Form gelungener Erinnerung als Voraussetzung für eine kritische Auseinandersetzung mit den im Archiv des individuellen und kollektiven Gedächtnisses gespeicherten Erfahrungen. Zugleich bildet sie im umfassenden Sinn die kritische Instanz, die den Wissenschaften - bei Ricœur: der Geschichtswissenschaft - ihren Platz im kulturellen Gedächtnis und den Prozessen kultureller Erinnerung zuweist. Voraussetzung für das Gelingen der Erinnerungsarbeit ist eine Haltung psychischer und kritisch-reflexiver Distanz zur Vergangenheit und zur eigenen Geschichte. Eine gelungene Erinnerungsarbeit setzt die Individuen instand, Verantwortung für ihr Handeln zu übernehmen, angetanes oder erlittenes Unrecht auszusprechen ohne es gegeneinander aufzurechnen. Erinnerungsarbeit setzt ein "rekonstruierendes Gedächtnis" voraus - das "wiederholende Gedächtnis« bleibt hingegen im Kreislauf von Schuld und Verdrängen, erlittenem Leid und Trauma, von Projektion und Vergessen verhaftet. ${ }^{14}$

Vergessen und Verdrängen sind Formen des wiederholenden Gedächtnisses. Während die Opfer ihre traumatischen Erfahrungen einkapselten und sprachlos wurden, projizierten die Täter eigene Schuldgefühle auf die Emigranten. Klaus Briegleb ${ }^{15}$ und Stefan Braese ${ }^{16}$ haben gezeigt, wie diese symbolische Ausgrenzung in der deutschen Nachkriegsliteratur zur faktischen Ausgrenzung, einem »Nach-Exil» deutschsprachiger jüdischer Autoren-Paul 
Celan, Wolfgang Hildesheimer, Peter Weiss oder Edgar Hilsenrath - geführt hat. Der Schuld- und Verdrängungszusammenhang, wie er im kommunikativen Gedächtnis entsteht, wirkt bis heute nach und ist eine der Ursachen für Defizite und Leerstellen im kulturellen Gedächtnis des Exils. Aber auch die Erinnerung und wissenschaftliche Aufarbeitung des Exils ist nicht frei von Momenten des wiederholenden Gedächtnisses. Konzepte etwa wie das "andere Deutschland" oder der "Antifaschismus", ästhetische Programme wie der "Realismus" haben, sofern sie nicht als geschichtliche Erinnerungsrahmen angesehen wurden, in der Forschung und öffentlichen Wahrnehmung die Funktion identifikatorischer Mythen übernommen, mit deren Hilfe das Exil, seine politischen und ästhetischen Programme und die sich daraus ergebenden historischen Erwartungen und Konfliktlinien affirmativ in die Gegenwart hineinverlängert wurden. ${ }^{17}$

Die Beiträge des vorliegenden Bandes orientieren sich am "rekonstruierenden Gedächtnis". Die Autorinnen und Autoren beziehen sich auf das Exil als "Archiv". Sie untersuchen den Stellenwert des Exils in einzelnen Gedächtnisfeldern-Archiven, Museen und Bibliotheken, Schulbüchern und literarischen Texten - und vermitteln einen Einblick in die Methoden und Formen der Erinnerungsarbeit. Gerhard Paul rekonstruiert in seinem Beitrag zur Rolle des politischen Exils in den deutschsprachigen Schulbüchern die Geschichte einer verhinderten Rezeption. Sein Hinweis auf "Leerstellen" im kulturellen Gedächtnis verweist auf Defizite und Desiderate der gegenwärtigen Erinnerungskultur, regt aber zugleich an, nach dem Stellenwert des Exils in anderen Bereichen der Erinnerungskultur zu fragen. Als einziges öffentliches Erinnerungsdatum an das Exil fungiert die Bücherverbrennung vom 1o. Mai 1933. Christoph Kopke und Werner Treß rekapitulieren deren Erinnerungsgeschichte und kommemorative Symbolik und beschreiben einen Vorgang, der die historische Tatsache geistiger Verfolgung und Zensur zum erinnerungspolitischen Schlüsselereignis des gesamten Exils gemacht hat.

Archive und Museen gehören zu den traditionellen Gedächtnisspeichern, die aus ihren Beständen organisierten Ausstellungen machen sie zu aktiven Erinnerungsorten. Von der "vermittelten Erinnerung" im Archiv und seinen Ausstellungen handelt der Beitrag von Sylvia Asmus und Brita Eckert zur Geschichte des "Deutsches Exilarchivs". Die bloße Präsentation von Dokumenten wird ersetzt durch visualisierende Verfahren wie die Rekonstruktion von Räumen, in denen Geschichte erfahren werden kann. Imaginäre Orte der Erinnerung und Erfahrung sind das Marbacher "Literaturmuseum der Moderne", dessen Konzeption von Heike Gfrereis und Verena Staack vorgestellt wird, und das "Museum der deutschsprachigen Juden« in Israel, dessen Geschichte Lena Kreppel im Kontext der Geschichte des israelischen Erinnerungsdiskurses darstellt. Die Verräumlichung der Erinnerung und ihre 
pädagogische Konkretisierung stellen Angelika Meyer, Marion Neumann und Sabine Hillebrecht am Beispiel der Ausstellung über Varian Fry in der Berliner Akademie der Künste vor. Heinz Högerle und Barbara Staudacher schildern am Beispiel des schwäbischen Dorfes Rexingen und Shavei Zion in Israel, wie das Projekt einer Ausstellung, die Zeugenbefragungen, Erinnerungsprotokolle und -archive und die Ausstellung selbst als "dritter Raum" zum Begegnungsort jüdischer Emigranten und deren Nachkommen mit den Einwohnern ihrer früheren Heimatgemeinde werden. - Gespächsprotokolle des Freundes- und Schülerkreises des Sozialpädagogen Ernst Papanek, die Inge Hansen-Schaberg aufgezeichnet hat, rekonstruieren Erinnerung als kommunikativen Raum.

Das rekonstruierende Gedächtnis hat auch konkrete historische Ursachen: Es verweist auf Brüche und Lücken der Tradierung als Folge gewaltsamer Prozesse der Vertreibung und Vernichtung von Menschen, der Enteignung ihres Besitzes, darunter auch des Raubs von Büchern und Kunstgegenständen nach 1933. Provenienzforschung ist heute ein zentraler Teil der öffentlichen und wissenschaftlichen Erinnerungsarbeit. Kunsthistoriker und Bibliothekswissenschaftler haben im Rahmen von Restitutionsansprüchen und Bestandsprüfungen erinnerungsgeschichtliche Methoden entwickelt, um die Spuren zu entziffern, die Aufschluss über die früheren Besitzer und das Schicksal von Kunstsammlungen und Bibliotheken geben. Regine Dehnel stellt Verfahren und Vorgehensweise der bibliothekswissenschaftlichen Provenienzforschung am Beispiel des "Projekts zur Suche nach geraubten Büchern" an der Gottfried Wilhelm Leibniz Bibliothek in Hannover dar; Anja Heuß verweist auf die erinnerungsgeschichtliche Bedeutung von Rechtsnormen und ihre Anwendung für die historische Wahrnehmung des Kunstraubs und die Restitution von Kunstwerken; Ines Rotermund-Reynard stellt am Beispiel der Geschichte der Kunstsammlung Paul Westheims die Methodik kunstgeschichtlicher Recherche vor.

Den Band beschließen zwei Beiträge, die den literarischen Narrativen rekonstruierender Erinnerung im Exil selbst nachgehen: Lutz Winckler untersucht die Erinnerungsarbeit in Anna Seghers Transit und Wulf Koepke meditiert am Beispiel von Heinrichs Manns Autobiografie Ein Zeitalter wird besichtigt über den Versuch, eine Lebensbilanz als erinnerungsgeschichtlichen Rückblick auf ein Zeitalter zu schreiben.

Die Frage nach dem Gedächtnis des Exils schließt die Frage des Vergessens ein. Die "kollektive Verdrängung", wie sie für die BRD der 1950er und 1960er Jahre galt, die parteilich orientierte, eingeschränkte Rezeption in der DDR haben zu Versäumnissen geführt, die unaufhebbar sind. Dem stehen die Bemühungen von Archiven, Wissenschaften, Verlagen und in jüngster Zeit von Kunstmuseen, wissenschaftlichen Bibliotheken und ihren wissenschaftlichen MitarbeiterInnen, von engagierten AusstellungsmacherInnen 
gegenüber, die Geschichte des politischen und kulturellen Exils aufzuarbeiten und dem öffentlichen Bewusstsein zugänglich zu machen. Für eine Bilanz des erinnerungskulturellen Stellenwerts des Exils erscheint es noch zu früh. Die Darstellung der Erinnerungsarbeit in verschiedenen kulturellen Gedächtnisfeldern, der Vergleich der unterschiedlichen Methoden und erinnerungskulturellen Narrative ${ }^{18}$ sind erste Schritte. Sinnvoll und notwendig erscheint das Nachdenken darüber, in welche Richtung sich die Erinnerungsarbeit entwickeln sollte.

Von Wertgemeinschaften zu Erinnerungsgemeinschaften - so könnte die Perspektive für eine kritische Erinnerungskultur, den Wandel der Exilforschung von einer am Zeugen orientierten zu einer am Archiv arbeitenden Wissenschaft lauten. Wertgemeinschaften sind geschlossen, auf die Werte der eigenen Gruppe bezogen, Erinnerungsgemeinschaften sind offen, die eigenen Werte mit anderen vergleichend: Ihr Verhältnis zur Geschichte ist rekonstruktiv nicht revokativ wie das der Wertgemeinschaften. Die Einstellung zur Gegenwart und Zukunft ist nicht vorgegeben, sondern offen - Werte und Ziele sind verhandelbar. Als ein Element, das in diese Verhandlung eingebracht wird, bleibt die Geschichte des Exils und die mit ihr verbundenen Erfahrungen, die Geschichte der Zeugen und ihrer Werke wichtig. Die Erinnerung daran gilt aber über das historische Exil hinaus allen, die vor oder nachher ein ähnliches Schicksal als "Migranten« betrifft.

1 Aleida Assmann: Zur "Metaphorik der Erinnerung . In: Aleida Assmann und Dietrich Harth (Hg.): Kulturals Lebenswelt und Monument. Frankfurt a. M. 1991, S. 11. - 2 Aleida Assmann: "Vier Formen des Gedächtnisses". In: Erwägen. Wissen. Ethik 13 (2002), S. $184 \mathrm{f}$. - 3 Zur Unterscheidung von kommunikativem und kulturellem Gedächtnis vgl. Jan Assmann: Das kulturelle Gedächtnis. Schrift, Erinnerung und politische Identität in frühen Hochkulturen. München 2000, S. 48 ff. und Aleida Assmann: Der lange Schatten der Vergangenheit. Erinnerungskultur und Geschichtspolitik. München 2006, S. 21-61, insbes. S. 54 der Versuch eines analytischen Vergleichsschemas. Einen Überblick über die Forschungs- und Begriffsgeschichte der Erinnerungskultur und Formen der medialen, insbesondere auch der literarischen Vermittlung gibt Astrid Erll: Kollektives Gedächtnis und Erinnerungskulturen. Eine Einführung. Stuttgart/Weimar 2005. - 4 Jan Assmann: „Kollektives Gedächtnis und kulturelle Identität". In: Jan Assmann und Tonio Hölscher (Hg.): Kultur und Gedächtnis. Frankfurt a. M. 1988, S. 12. — 5 Assmann: "Vier Formen des Gedächtnisses" (s. Anm. 2), S. 189. - 6 Assmann: "Kollektives Gedächtnis und kulturelle Identität" (s. Anm. 4 ), S. 15. - 7 Maurice Halbwachs: Les cadres sociaux de la mémoire. Paris 1952. Hier zitiert nach der deutschen Ausgabe: Das Gedächtnis und seine sozialen Bedingungen. Frankfurt a. M. 1985, S. $125 \mathrm{ff}$. 8 Paul Ricceur: La Mémoire, L'Histoire, L'Oubli. Paris 2000, S. 181 ff. - 9 Ebd., S. 214 f. 10 Krzysztof Pomian: Der Ursprung des Museums. Berlin 1993, S. 46 ff. spricht von Semioforen (S. 46 ff.) Er versteht darunter Gegenstände, deren Materialität ihrer Zeichenfunktion untergeordnet ist. Es handelt sich um wnatürliche oder künstlerische Gegenstände, die zeitweise oder endgültig aus dem Kreislauf ökonomischer Aktivitäten herausgehalten werden, und (...) an einem abgeschlossenen Ort (...) ausgesellt (...) und angesehen werden können" 


\section{Vorwort}

(S. 16). Zu diesen Orten zählen Museen, Bibliotheken und Archive. - 11 Günter Oesterle: "Souvenir und Andenken". In: Der Souvenir. Erinnerung in Dingen von der Reliquie zum Andenken. Museum für Kommunikation Frankfurt a. M. 2006, S.31. - 12 Ulla Langkau-Alex: "Geschichte der Exilforschung « und Heinz Boberach: "Quellen zur Exilforschung “. In: Claus-Dieter Krohn, Patrik von zur Mühlen, Gerhard Paul und Lutz Winckler (Hg.): Handbuch der deutschsprachigen Emigration 1933-1945. Darmstadt 1998, S. 1195 ff. und 1209 ff. - 13 Paul Ricceur: Das Rätsel der Vergangenheit. Erinnern - Vergessen - Verzeihen. Göttingen 2000, S. 100 ff. - 14 Ebd., S. 129. - Zur Herleitung und Funktion der Begriffe mémoire-répétition und mémoire critique vgl. auch ders.: La Mémoire, L' Histoire, L'Oubli (s. Anm. 8 ), S. 82 ff. - 15 Klaus Briegleb: Mißachtung und Tabu. Eine Streitschrift zur Frage: , Wie antisemitisch war die Gruppe 47?, Berlin/Wien 2003 spricht von weiner gescheiterten Gedächtnisgeschichte nach 1945 «(S.77). - 16 Stephan Braese: Die andere Erinnerung. Jüdische Autoren in der westdeutschen Literatur. Berlin/Wien 2001. - 17 Lutz Winckler: "Mythen der Exilforschung? « In: Exilforschung. Ein internationales Jahrbuch. Claus-Dieter Krohn, Erwin Rotermund, Lutz Winckler und Wulf Koepke (Hg.), Bd. 13 (1995), S. 68 ff. - 18 Hinweise für literarische Narrative finden sich bei Erll: Kollektives Gedächtnis und Erinnerungskulturen (s. Anm. 3), S. 149 ff. 